\title{
Promising results using a simple rehabilitation program to treat partial ruptures in the Achilles midportion
}

\author{
Lorenzo Anthony Masci, Hakan Alfredson \\ Pure Sports Medicine, London, UK \\ Correspondence: Lorenzo Anthony Masci. Address: Pure Sports Medicine, 116 Cromwell Rd London W86J N, UK. Email: \\ Lorenzo.masci@puresportsmed.com. \\ Received: February 4, 2013 \\ DOI : $10.5430 / j b g c . v 3 n 4 p 47$ \\ Accepted: March 14, 2013 \\ Online Published: July 10, 2013 \\ URL: http://dx.doi.org/10.5430/jbgc.v3n4p47
}

\begin{abstract}
Objectives: To assess the outcome of a conservative rehabilitation program in treating partial ruptures in the Achilles midportion.

Materials and methods: Twenty-six consecutive recreational and elite-level athletes (22 men and 4 women) with an ultrasound and Doppler-verified partial rupture in the Achilles midportion were followed prospectively during treatments with heel lifts, modified activity level and avoidance of calf stretching exercises for 3 months.

Clinical examination, ultrasound (US) with Colour Doppler (DP) examination and pain scores during rest and walking (VAS scores) were assessed at baseline and 3 months after instituted intervention. Patients were followed up by a telephone interview at 6 months.

Results: At the 3 months follow-up, there were significantly reduced VAS scores at rest $(p=0.018)$ and walking $(p=$ 0.014 ), and improvement in the US+DP findings in $25 / 26$ patients. One patient required surgery due to ongoing pain during activity and no tendon healing on US+DP. The 6-month follow-up revealed that 25/26 patients were satisfied with the treatment result.
\end{abstract}

Conclusion: A simple rehabilitation program seems successful at treating partial tears in the Achilles mid-portion.

\section{Key words}

Simple rehabilitation, Ultrasound, Partial Achilles tears

\section{I ntroduction}

Complete Achilles tendon ruptures are common among recreational and elite athletes ${ }^{[1]}$. The diagnosis of a complete rupture is often, but not always, diagnosed early and there are many scientific papers purporting the pros and cons of conservative versus surgical management ${ }^{[2,3]}$. On the contrary, there is a paucity of scientific literature regarding diagnosis and treatment of partial Achilles tendon tears. 
Early diagnosis of a partial tear can be difficult. The clinical presentation is thought to be unreliable, and imaging findings have been poorly described. Previously, there was little consensus as to the best modality for diagnosis of partial tears ${ }^{[4,5]}$. However, a recent study ${ }^{[6]}$ found specific ultrasound changes that correlated closely with macroscopic appearances from surgery. These specific changes include irregularity of tendon structure on the posterior (skin) side of the tendon with disruption of the posterior tendon fibers. In addition, Colour Doppler examination revealed high blood flow within the region of tendon discontinuity.

Unlike full-thickness tendon tears, the treatment of partial tears has not been adequately described. There are only a few, and relatively old, studies on surgical treatment of partial Achilles tendon ruptures ${ }^{[7-9]}$. However, the diagnosis of a partial tear in those studies was based on the macroscopic surgical findings of tendinopathy, and the treatment was excision of the pathologic or degenerative tendinopathy. These studies did not include partial tears diagnosed on ultrasound findings.

The aim of this study was to assess the outcome of a conservative treatment program on subjects with an ultrasound (US) with Colour Doppler (CP) diagnosed (6) partial tear in the Achilles mid-portion.

\section{Materials and methods}

A prospective study was performed on consecutive patients recruited at the Pure Sports Medicine Clinic in London, England. All patients were referred by doctors or physiotherapists for assessment of Achilles tendon pain.

Altogether, 26 patients (22 men and 4 women) with a mean age of 47.5 years (range 23-81) were recruited in the study. The mean duration of Achilles pain was 8 months (range 1.5-72 months).

Activity levels included running ( $\mathrm{n}=8)$, walking $(\mathrm{n}=5)$, tennis $(\mathrm{n}=2)$, rugby $(\mathrm{n}=2)$, triathlon $(\mathrm{n}=2)$, football $(\mathrm{n}=2)$, decathlon $(n=1)$, ballet $(n=1)$, gym $(n=1)$, squash $(n=1)$ and no activity $(n=1)$. There were 5 elite athletes: two from rugby, one from football, one from ballet and one from decathlon.

To be included in the study, patients required the following criteria:

1) Midportion Achilles tendon pain.

2) Ultrasound (US) with Colour Doppler (CD) findings of a partial tear on the posterior (skin) side of a thickened Achilles midportion ${ }^{[6]}$.

Eleven (42.3\%) patients had a history of sudden onset of pain while fifteen (57.7\%) had an insidious onset. Twelve (46.2\%) patients had a subjective feeling of leg or calf weakness.

Previous treatments are outlined in Table 1. Twenty (76.9\%) patients had been diagnosed with tendinopathy and had been treated with eccentric calf drops, and 15 (57.7\%) patients had been treated with an injection or combinations of injections including cortisone, autologous blood or high volume stripping (cortisone, marcaine and normal saline). Twenty-two (84.6\%) patients had had a previous ultrasound (15/26) and/or MRI (13/26) investigation for diagnosis. Nineteen (73.1\%) patients with a partial tear had co-existing tendinopathy, while 7 (26.9\%) had no co-existing tendinopathy on ultrasound with Colour Doppler examination.

Table 1. Previous treatments and Numberof subjects

\begin{tabular}{ll}
\hline Previous treatments & Number of subjects \\
\hline Eccentric exercises & 20 \\
Injections (blood, cortisone, high volume stripping) & 15 \\
Shock wave & 3 \\
Heel raises & 2 \\
\hline
\end{tabular}




\subsection{Ultrasound examination}

Ultrasound (US) with Colour Doppler (CD) examination was performed by the same experienced doctor (H.A.) in all patients using a linear multi-frequency (8-13MHz) transducer (Antares; Siemens). The Achilles tendon was examined with subjects in a prone position with their heels overhanging the examination table.

The specific ultrasound (US) with Colour Doppler (CD) findings ${ }^{[6]}$ of a partial Achilles tendon tear included (see Figure 1 and Figure 2):

1) Thickening of the Achilles tendon midportion (> 6mm)

2) Irregular and disrupted posterior (skin side) tendon fibers

3) Irregularity or disruption of the posterior tendon extending into the midportion

4) High blood flow in the region with tendon changes

Figure 1. US scan, longitudinal view, showing irregular and disrupted posterior tendon.

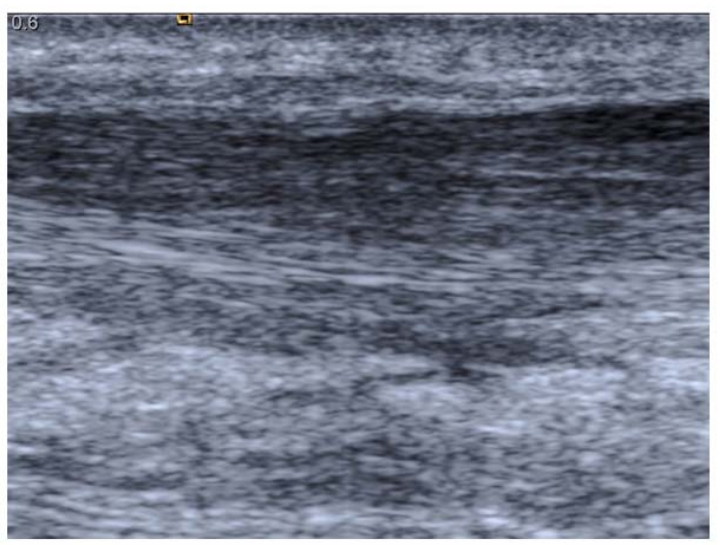

Figure 2. US+DP scan, longitudinal view, showing a thickened Achilles midportion, and irregular and disrupted posterior tendon with high blood flow.

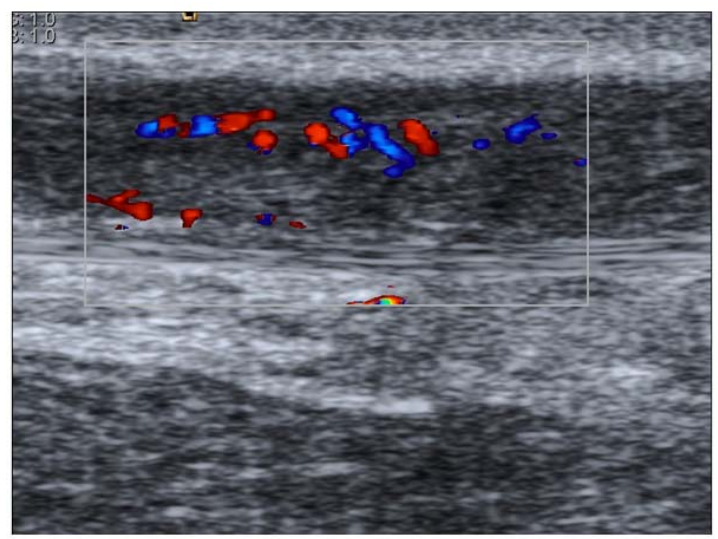

\subsection{Rehabilitation program}

All patients underwent a structured 3-month rehabilitation program:

1) 0-6 weeks: $2 \mathrm{~cm}$ heel lifts, avoid calf muscle stretching activity but walking, cycling and swimming allowed

2) 7-12 weeks: reduce heel lifts to $1 \mathrm{~cm}$, concentric calf raises beginning with seated calf raises $3 \times 15$ repetitions daily progressing to standing bilateral calf raises (from floor level to tip-toe) $3 \times 15$ reps daily, and then to single calf raises (from floor level to tip-toe) $3 \times 15$ daily. The exercises were performed 3 times/week together with cross training including walking, swimming and cycling. 
After the 3-month program, pain-free patients were told to remove the heel lifts, start with $3 \times 15$ reps of eccentric heel drops from tip toe to below floor level 3 times/week, and to gradually return to their previous (pre-injury) tendon loading activity.

\section{Outcome measures:}

1) Pain at rest and during walking. Patients recorded tendon pain at rest and during walking, initially and at 3 months. Pain was recorded on a 100mm long visual analogue scale (VAS) where no pain was recorded as 0 and maximal pain was recorded as 100 .

2) Patient satisfaction (satisfied or not satisfied). Via a telephone interview six months after instigation of treatment, patients were interviewed and asked whether they had returned to their previous (pre-injury) activity (yes or no). Patient satisfaction was determined by self-reporting (satisfied or not satisfied).

\subsection{Statistics}

SPSS version 19.0 was used for statistical calculations.

Wilcoxon signed rank-test was used to analyse differences in mean VAS scores pre- and post-treatment. Differences were considered significant at $p<0.05$.

Patient consent was obtained before inclusion in the study. This study met the ethical standards of the International Journal of Sports Medicine ${ }^{[10]}$.

\section{Results}

Before treatment, patients recorded pain at rest (mean VAS 13.96, range 0 to 50) and during walking (mean VAS 53.93, range 20.1 to 90.3). After 3 months of treatment, there was a significantly reduced mean VAS at rest from 13.96 to 2.63 ( $\mathrm{SD}=10.25, p=0.018$ ) and during walking from 53.93 to $15.1(\mathrm{SD}=20.28, p=0.014)$. One patient required surgery at Umea, Sweden at 3 months due to ongoing pain at rest and during walking, and US+CD findings of poor healing of the tendon tear.

In 25/26 patients, there was improvement in the US+CD findings at 3 months. Improvement was defined as improved collagen fibre alignment and normalisation of the echogenicity of the partial tear, and reduced or absent blood flow within the partial tear.

The 6-month telephone follow-up revealed that 25/26 patients were satisfied with the treatment, and 23/26 patients were back at previous (pre-injury) activity. Two patients had not returned to their chosen sport, but this was due to the development of other injuries unrelated to their Achilles tendon rupture. As stated above, one patient had surgery at 3 months and was undergoing rehabilitation at 6 months.

Decreased pain during rest and activity correlated with improvement in the US+CD findings at 3 months

\section{Discussion}

The typical presentation of a partial rupture is said to be similar to a complete rupture. The history is usually sudden onset of Achilles or calf pain following an explosive manoeuvre in addition to evidence of clinical lengthening ${ }^{[5]}$. One may therefore surmise that the diagnosis of a partial tear could be made adequately on clinical presentation without the need for imaging. However, unlike complete tears, there is little evidence to suggest that the diagnosis of partial tears can be made on clinical grounds. Alfredson et al. ${ }^{[6]}$ found that only 14 out of 17 of patients with a partial rupture had acute onset of pain 
and only 7 out of 17 patients had evidence of tendon lengthening on examination. Similarly, in the current study, we found that only 11 (42.3\%) out of 26 patients had a history of sudden Achilles tendon pain. Therefore, the results of these two studies taken together would suggest that the diagnosis of a partial tear cannot reliably be made on clinical grounds and requires imaging. Moreover, we believe that the investigation of choice should be ultrasound, as this imaging modality has been shown to differentiate partial tears from other causes such as tendinopathy ${ }^{[6]}$. In this study and the previous study ${ }^{[6]}$, we found that MRI missed a considerable number of partial tears diagnosed by this new ultrasound-defined definition ${ }^{[6]}$. We would argue, therefore, that MRI is less superior than ultrasound at diagnosing partial tears. More studies are required to compare ultrasound to MRI in diagnosing partial tears.

Rupture of Achilles tendons has been associated with numerous conditions including previous degeneration of the tendon ${ }^{[11]}$, fluoroquinolone use ${ }^{[12]}$ and corticosteroid administration ${ }^{[13]}$. In the current study, ten (38.46\%) patients had one or more injections prior to the diagnosis of partial Achilles rupture. These injections included corticosteroid, autologous blood and high volume stripping (consisting of cortisone, marcaine and normal saline). The high incidence of injections could suggest that there is an association between injections and partial tendon tears. Previous studies have implicated corticosteroid administration in the aetiology of tendon disruption. One study ${ }^{[14]}$ revealed that injection of corticosteroid into rabbit tendons led to necrosis within 45 minutes. Another study ${ }^{[15]}$ demonstrated significant biomechanical weakening of tendons for up to 14 days after intra-tendinous corticosteroid injections. While there are no studies linking autologous blood and high volume stripping to partial tears, further studies are required to investigate a possible association.

It could possibly be argued that the patients in this study did not have partial ruptures, but rather tendinopathy (thickening, irregular structure, vascular ingrowth). However, although many of these tendons also showed tendinopathy, the US+CD findings clearly showed changes on the posterior side (skin side) of the tendon; defined as a partial rupture in a previous study ${ }^{[6]}$, where US+CD findings were correlated with macroscopic surgical findings of a partial rupture on the posterior side (most loaded side) of the tendon. Tendinopathy alone is correlated with abnormalities mainly located on the less loaded ventral side (resting side) of the tendon. Moreover, studies suggest that heavy tendon loading is the optimal conservative treatment for tendinopathy ${ }^{[6]}$, an approach that is more or less opposite to the one given in the current study. The fact that the patients in this study improved with rest and avoidance of stretching suggest that the changes on ultrasound were not tendinopathy.

This study demonstrates that tendinopathy and partial ruptures may co-exist. As the treatment of these two conditions are completely different, eccentric training for tendinopathy and avoidance of stretching/lengthening of the tendon for a partial rupture, it is important to obtain an accurate diagnosis. This premise is reinforced in the current study where 20/26 patients had been prescribed eccentric training as treatment before their diagnosis of a partial tendon tear. Potentially treating a partial tear with tendon loading may lead to extension of the tear and tendon lengthening, a notoriously difficult condition to treat ${ }^{[11]}$.

The results of this study suggest that a relatively simple rehabilitation program including heel lifts, a modified activity level and avoidance of calf stretching exercises is an effective treatment for partial tears in the Achilles tendon midportion. There were significantly reduced pain levels at rest and activity in 25/26 patients, and improvement in US+CD findings suggestive of healing. Although we cannot be absolutely sure that ruptures had healed at the 3-month follow-up, the reduced tendon pain in conjunction with improvement of tendon structure and normalisation of blood flow on the posterior (skin side) of the tendon suggests satisfactory healing. Furthermore, the fact that the majority of patients following treatment could return to their previous activity with 25/26 patients being satisfied with the result of treatment at the 6 month follow-up lends credence to the success of this conservative program.

It could be argued that the 3 months relative rest, and not the designed treatment protocol, was responsible for the good clinical results. However, this is unlikely since the majority of the patients had had a long duration of pain symptoms 
(mean 8 months) and had tried long periods of rest without effect on the tendon pain. Therefore, we strongly believe that it is the avoidance of tendon stretching/lengthening, by using heel lifts and avoiding all kinds of tendon stretching activities, which is the key factor for healing of the partial rupture.

Only one patient out of 26 required surgical treatment after undergoing the rehabilitation program. This result suggests that partial tears of Achilles tendons respond well to avoidance of stretching/lengthening activities, obviating the need for surgery in most cases. The one patient that did not respond to the rehabilitation program had been treated with multiple injections, including autologous blood and high volume stripping (consisting of marcaine, saline and cortisone). At surgery, there was extensive necrotic tissue within the posterior tendon (skin side) requiring debridement. This could possibly explain the poor result from the rehabilitation program. However, nine other patients also had had injection treatments and responded well to the rehabilitation program. Hypothetically, it could be postulated that one patient requiring surgical treatment had been exposed to an intra-tendinous injection leading to local tendon necrosis while the other patients had not.

There are important limitations to this study. Firstly, the most optimal design for evaluating therapeautic interventions would have been a randomized controlled study. However, it is difficult to ethically justify a study where patients that already have had a long period of symptoms (mean 8 months) can be randomized to another 6 months of rest. Secondly, this study contained relatively small patient numbers. Thirdly, the follow-up time was relatively short. However, it could be argued that the follow-up time was satisfactory for an acute injury with an estimated healing time of 3-4 months. Consequently, a 3-month clinical and US+CD follow-up together with a 6-month telephone interview should be satisfactory to establish if there was adequate healing for a return to pain-free Achilles tendon loading activities. Fourthly, the patients in this study had different activity levels with dissimilar baseline characteristics; but the majority were involved in activities that placed high loads on their Achilles tendons. We believe that the good results demonstrated at the 3- and 6-month follow-up in these patients indicate satisfactory healing of the partial ruptures. Nevertheless, further research is required to substantiate the short-term positive effects of this type of conservative treatment program, and to confirm that these outcomes are maintained in the long term.

This rehabilitation program consisted of 3 months of rehabilitation before heavy loading and return to sports-specific activity. Although the results from this program were satisfactory, one could argue that a 3-month rehabilitation program is perhaps too long, particularly for elite athletes. On the other hand, 3 months healing time seems appropriate for tendon tissue. Although not documented in the results of the study, a number of subjects in this study reported significant improvement in pain and function after only 6 weeks of conservative treatment. It could be argued that patients may have been able to return to full function earlier than three months. The size of the partial rupture is most likely of significant importance for healing time. Further research is required to define the rupture size in relation to healing time.

In conclusion, the short-term results of this study demonstrate that a rehabilitation program focusing on avoiding stretching/lengthening of the Achilles is effective at treating US+CD-diagnosed partial midportion tears. The study also demonstrates that the diagnosis of partial tears cannot be reliably made on clinical grounds alone, but requires further imaging such as high resolution US+DP.

\section{References}

[1] Kvist M. Achilles tendon injuries in athletes. Sports Med. 1994; 18(3): 173-201. PMid:7809555

http://dx.doi.org/10.2165/00007256-199418030-00004

[2] Nilsson Helander K. Acute Achilles tendon rupture-Evaluation of treatment and complications. Thesis. University of Gothenburg ISBN 978-91-628-7720-0 2009.

[3] Bhandari M, Guyatt GH, Siddiqui F, Morrow F, Busse J, Leighton RK, Sprague S, Schemitsch EH. Treatment of acute Achilles tendon ruptures: a systematic overview and meta-analysis. Clin Orthop Relat Res. 2002(400): 190-200. PMid:12072762

http://dx.doi.org/10.1097/00003086-200207000-00024 
[4] Kalebo P, Allenmark C, Peterson L, Sward L. Diagnostic value of ultrasonography in partial ruptures of the Achilles tendon. Am J Sports Med. 1992; 20(4): 378-381. PMid:1415877 http://dx.doi.org/10.1177/036354659202000402

[5] Kayser R, Mahlfeldt K, Heyde CE. Partial rupture of the proximal Achilles tendon: a differential diagnostic problem in ultrasound imaging. Br J Sports Med. 2005; 39(11): 838-842. PMid:16244194 http://dx.doi.org/10.1136/bjsm.2005.018416

[6] Alfredson H, Masci L, Ohberg. Partial mid-portion tendon ruptures: new sonographic findings helpful for diagnosis. Br J Sports Med. 2011; 45: 429-432. PMid:19945976 http://dx.doi.org/10.1136/bjsm.2009.067298

[7] Denstad TF, Roaas A. Surgical treatment of partial Achilles tendon rupture. Am J Sports Med. 1979 Jan-Feb; 7(1): 15-17. PMid:420383 http://dx.doi.org/10.1177/036354657900700104

[8] Ljungkvist R. Subcutaneous partial rupture of the Achilles tendon. Acta Orthop Scand. 1967; Suppl 113: 1.

[9] Morberg P et al. Long-term results after surgical treatment of partial Achilles tendon ruptures. Scand J Med Sci Sports. 1997; 7(5): 170-175.

[10] Harrison DJ, Atkinson G. International Journal of Sports Medicine - ethical standards in sport and exercise science research. Int J Sports Med. 2009; 30(10): 701-2. PMid:19809942 http://dx.doi.org/10.1055/s-0029-1237378

[11] Gabel S, Manoli A. Neglected rupture of the Achilles tendon. Foot Ankle Int. 1994; 15: 512-517. PMid:7820247 http://dx.doi.org/10.1177/107110079401500912

[12] Royer R J, Pierfitte C, Netter P. Features of tendon disorders and fluroquinolones. Therapie. 1994; 49: 75-6. PMid:8091374

[13] Unverfirth L J, Olix M L. The effect of local steroid injection on tendon. J Bone Joint Surg. 1972; 55-A: 1315.

[14] Balasubramaniam P, Prathap K. The effect of injection of hydrocortisone into rabbit calcaneal tendons. J Bone J Surg. 1972; 54-B: 729-734.

[15] Kennedy J C, Baxter Willis R. The effects of local steroid injections on tendons: a biomechanical and microscopic correlative study. Am J Sports Med. 1976; 4: 11-13. PMid:937623 http://dx.doi.org/10.1177/036354657600400103 